\title{
Tangence
}

\section{Écritures marginales en France}

Être écrivain d'origine maghrébine

\section{Azouz Begag}

Numéro 59, janvier 1999

Écrivains d'ailleurs

URI : https://id.erudit.org/iderudit/025992ar

DOI : https://doi.org/10.7202/025992ar

Aller au sommaire du numéro

Éditeur(s)

Tangence

ISSN

0226-9554 (imprimé)

1710-0305 (numérique)

Découvrir la revue

Citer cet article

Begag, A. (1999). Écritures marginales en France : être écrivain d'origine

maghrébine. Tangence, (59), 62-76. https://doi.org/10.7202/025992ar d'utilisation que vous pouvez consulter en ligne.

https://apropos.erudit.org/fr/usagers/politique-dutilisation/ 


\section{Écritures marginales en France. Être écrivain d'origine maghrébine}

\section{Azouz Begag}

En France, depuis la crise économique de la fin des années soixante-dix, autour des grandes villes, dans les quartiers d'habitat social, le visage de la société a changé. Vue depuis ses frontières intérieures, la France est bien une société multiculturelle dans laquelle les populations issues de l'immigration africaine, et maghrébine en particulier, subissent le plus de difficultés économiques et sociales. Cette réalité interroge l'efficacité de la tradition française d'intégration. En effet, dans ces quartiers en difficultés des revendications à caractère ethno-communautaire émergent et dérogent radicalement à la tradition française issue de la Révolution. Certes, on ne peut douter du caractère généreux de la philosophie de la mixité sur laquelle repose le modèle français laïc et républicain, mais on doit reconnaître qu'aujourd'hui il subit de graves revers causés par l'exclusion économique et sociale. Dans l'esprit français de l'intégration citoyenne, l'école revêt une fonction de creuset de populations de toute importance. Or, aujourd'hui, dans les établissements où sont scolarisés de nombreux enfants d'immigrés originaires d'Afrique, souvent majoritairement, les professeurs de français peuvent-ils continuer à enseigner Molière ou Maupassant comme il y a vingt ans?

Parmi les outils de lutte contre l'exclusion sociale, la lecture, entendue comme l'accès des enfants et des jeunes au monde de la curiosité, du questionnement, est fondamental. C'est pour cette raison que mon engagement social depuis plus de dix ans vise à promouvoir la lecture dans les milieux défavorisés, immigrés en particulier, des banlieues d'habitat social. Mais de quelle promotion s'agit-il? Pratiquement, quels livres faut-il présenter à des jeunes d'origine maghrébine de milieu modeste, pour lesquels lire, loin d'être un acte spontané, est plutôt rebutant?

Certes, il existe la littérature maghrébine d'expression française, représentée par les écrivains tels Tahar Ben Jelloun, Driss Chraibi, Rachid Mimouni, Mohamed Dib, Kateb Yacine ${ }^{1}$... mais

1 Bonn, Charles (dir), Antbologie de la littérature algérienne (1950-1987), Paris, Le Livre de poche, 1990. 
elle est peu connue dans les établissements scolaires. Du reste, le public que constituent les enfants de l'immigration maghrébine en France n'est pas celui auquel s'adresse cette littérature. Ici, les jeunes et les auteurs ne s'identifient pas aux mêmes temps historiques, ne parlent pas les mêmes mots, ne vivent pas les mêmes réalités sociales. En bref, un effet générationnel s'incruste ici entre les deux groupes. Quand Ben Jelloun et Chrabi écrivent sur le Maroc, Mimouni et Dib sur l'Algérie, Meddeb et Bekri sur la Tunisie, leurs écritures ne trouvent pas écho dans l'identité des jeunes d'origine maghrébine en France.

Depuis une dizaine d'années, mon activité de chercheur en sciences sociales et d'écrivain "beur " me mène à la rencontre des collégiens, âgés généralement entre 13 et 16 ans dans les quartiers en difficultés de toutes les villes de France. En tant qu'auteur, spécialisé dans la littérature "pour la jeunesse", né à Lyon, de parents immigrés algériens, ayant réalisé une intégration dans la société française via le système éducatif, ma présence dans ces établissements scolaires où étudient de nombreux enfants de l'immigration maghrébine prend un sens hautement symbolique ${ }^{2}$, car bien souvent, c'est le rapport de tous ces élèves "non blancs" à la lecture, à l'école, à la classe, aux enseignants et donc à la société d'accueil qui est bouleversée. Ces écritures marginales qu'on appelle "littérature beur" présentent aujourd'hui un caractère novateur par rapport à la pédagogie de la lecture dans les milieux défavorisés ${ }^{3}$.

\section{La langue comme revendication de l'exclusion}

Globalement, dans les établissements scolaires où je suis invité à rencontrer mes jeunes lecteurs, le niveau scolaire d'une majorité d'élèves, plutôt faible, témoigne de la situation socio-économique des familles qui habitent dans les cités. Ce constat va de pair avec une pratique de la lecture quasi inexistante, dénuée de plaisir. Peu d'élèves lisent librement, car l'acte de lire est associé à une

2 Voir à ce propos la thèse de Enrica Govoni, Uno scrittore arabo/francese di "seconda generazione": Azouz Begag e il tema dell'identità culturale, tesi di Laurea in Lingua e Letteratura Francese, Università degli studi di Bologna, Facoltà du Magistero, Corso di Laurea in Lingue e Letterature Straniere, 19931994.

3 Hargreaves Alec G., Immigration and Identity in Beur Fiction. Voices from the North African Community in France, Oxford, Berg, 1997. 
64

obligation scolaire, un devoir. Il ne revêt guère d'utilité consciente, implique une trop grande concentration, en raison notamment de la difficulté de reconnaissance des mots méconnus, obscurs, incompréhensibles pour les élèves. Pour les enfants issus de milieux populaires, le livre véhicule également l'image de leur propre échec scolaire et constitue le signe de ce qui est inaccessible ou interdit. Il est d'autant plus rejeté qu'il renvoie une vision édulcorée de la réalité sociale de leur cité, où très tôt ils acquièrent une identité de la marge, dont l'usage d'une langue spécifique est l'un des facteurs les plus marquants de ces dernières années.

En France, cela fait déjà plusieurs années que des recherches explorent ce qui est communément appelé le langage des banlieues comme un nouveau phénomène socio-urbain ${ }^{4}$. En ce qui me concerne, je ne dissocie pas les pratiques langagières des jeunes des banlieues de la problématique générale de leur exclusion de la société française. Depuis une dizaine d'années en effet, je mène des ateliers d'écritures dans des classes de collèges des quartiers d'habitat social, dans les cités périphériques et je constate que beaucoup de textes écrits par les élèves (souvent d'origine immigrée) révèlent de sérieux problèmes de maîtrise de la langue française officielle dans ces quartiers. À terme, même les apprentissages élémentaires de la langue commune risquent d'être méconnus dans ces zones urbaines défavorisées, ajoutant ainsi une stigmatisation supplémentaire pour des jeunes qui sont déjà pénalisés dans leur accès à la société par leur adresse ${ }^{5}$ et/ou leur faciès. L'appauvrissement considérable de la maîtrise des codes sociaux de la communication (la fracture linguistique) que nous constatons reflète ainsi l'emprisonnement progressif des jeunes d'origine modeste dans la réclusion sociale et territoriale ${ }^{6}$.

Le parler des jeunes des banlieues, médiatisé par le théâtre populaire "Smaïn", la télévision "Les Guignols de l'info", la radio,

4 Voir à titre d'illustration le numéro de la revue Écarts d'identité (Grenoble) consacré à "La langue des autres. Immigrations et pratiques langagières", $\mathrm{n}^{\circ} 76$, mars 1997.

5 Lorsque la cité où ils habitent souffre d'une mauvaise réputation dans le reste de la ville, cela constitue un handicap pour la recherche d'emploi, par exemple, l'employeur étant influencé négativement par les préjugés...

$6 C f$. Le très instructif dictionnaire de Jean-Pierre Goudailler, Comment tu tchatches! Dictionnaire du français contemporain des cités, Paris, Maisonneuve et Larose, 1997 et Jean-Michen Déugis et Aziz Zemouri, Parlers de banlieue, Paris, Plon, 1996. 
le cinéma, est peut-être le symptôme d'une école, socle de la formation du citoyen français, qui n'aurait plus la capacité d'assurer sa mission d'apprentissage des codes minimums de la communication sociale.

L'histoire des jeunes des cités s'écrit souvent à l'extérieur des cités et aux dépens de leurs habitants. Il y a une espèce d'usurpation de l'existence des autres, de leur originalité, de leur spontanéité, de leur vulnérabilité, qui est gênante, et je voudrais citer en exemple la lecture de ce roman Lila dit ça, paru au printemps 1996, sous la signature d'un certain Chimo, dont on ne saurait pas s'il est locataire d'un HLM en banlieue parisienne ou directeur d'une maison d'édition boulevard Saint-Germain. Le lieu du roman n'est pas choisi au hasard: Lila, l'héroïne, habite dans une cité "hostile" (l'amour au milieu de la désolation) et l'éditeur s'évertue à en faire un atout exotique pour évoquer en quatrième de couverture "ce livre naif et sophistiqué, cette fleur poussée sur le béton des cités. On peut parier que l'auteur n'est pas un enfant des banlieues, un inconnu surdoué, mais plutôt un écrivain "confirmé" qui sait tirer profit (au sens commercial du terme) d'un effet de mode sur les banlieues. Exactement de la même manière, on peut citer un roman publié en septembre 1997 aux éditions Balland à Paris, Vivre me tue, et qui a été présenté comme l'œuvre d'un certain Paul Smaïl, jeune "beur" francomarocain, qui "ne veut être jugé qu'au travers de ses écrits" et que de ce fait personne n'a jamais vu. Là encore, il y a eu une confusion littéraro-commerciale derrière ce livre qui a connu un très gros succès de librairie ce dernier trimestre 1997.

En extrapolant ces exemples, on peut même remarquer que ce sont rarement les jeunes des cités eux-mêmes qui sont à la base de l'autobiographie de leur quartier, de la médiatisation de leur vie sociale (le film de Malik Chibane, Hexagone, sorti en 1994, est l'un des rares exemples au cinéma). Ce sont en effet toujours des chercheurs de l'extérieur, journalistes, qui, à l'occasion de reportages, d'enquêtes, d'observation sur le terrain, se nourrissent des particularismes repérés dans les cités, des phénomènes socio-culturels novateurs.

\section{Clichés et fausses pistes}

S'il faut manipuler avec précaution les expressions telles que Jeunes des banlieues ou Langue des banlieues pour éviter des 
66

amalgames trompeurs, en revanche, on peut confirmer l'existence d'un esprit "périphérique" qui se définit, via la médiatisation du phénomène banlieue, en référence, souvent réactive, à la socité centrale, au centre-ville, au pouvoir central, à l'ordre établi.

Le langage des jeunes de banlieue est-il spécifique à cette population, à leur situation sociale, géographique, ou bien renvoit-il de manière plus générale à d'autres populations, d'autres situations temporelles, sociales et géographiques? Se référer à l'argot populaire français des années soixante ou à "la langue des Gitans ", très présente dans les expressions des jeunes de banlieues, peut aider à décentrer la problématique et à s'interroger sur les modalités d'adoption, d'usage et de détournement d'une langue nationale par des individus ou des groupes appartenant à une minorité ethnique, par exemple. À cet égard, il est étonnant de remarquer les très nombreux emprunts des mots de banlieues à la langue des Gitans: c'est le cas de ceux qui se terminent par ave comme chourave (voler), marave (se battre), dicave (regarder), nachave (s'en aller), balnave (mentir)... Il est utile de connaître ces dérives sémantiques pour comprendre par exemple l'expression Arrête de m'balnave! que m'a servie un jour un jeune dans un bar, alors qu'il était en état d'ivresse. Il voulait me dire Cesse de me prendre pour un idiot avec tes sornettes!

Certains analystes voudraient présenter la langue des jeunes des cités défavorisées comme la marque d'un dynamisme, d'une créativité réconfortante. J'ai un avis contraire. Le constat que je fais après de nombreux ateliers d'écriture dans les cités, des rencontres littéraires dans les collèges depuis une dizaine d'années... est celui d'un appauvrissement considérable de la maîtrise des codes sociaux de la communication, qui emprisonne un peu plus les jeunes d'origine modeste dans l'exclusion sociale et territoriale.

\section{Espace de confusion}

On ne peut pas nier que dans les quartiers périphériques les codes linguistiques présentent des particularismes par rapport à ceux qui sont en vigueur dans des quartiers moins pauvres. L'origine ethnique (maghrébine ou africaine) des jeunes les conduit souvent à s'exprimer à la maison avec leurs parents dans une langue maternelle différente du français et, partant, à déformer, 
reformer, jouer avec la langue officielle apprise à l'école ${ }^{7}$. Or, lorsqu'on puise son langage parlé dans deux registres linguistiques différents, l'arabe dialectal et le français par exemple, sans en maîtriser aucun suffisamment, des confusions apparaissent inéluctablement. C'est dans cette zone d'interférence que se nourrit le parler des banlieues, zone de confusion qui est aussi un espace de malaise, de broyage et de brouillage. L'usage de codes linguistiques internes est également influencé par l'identité groupale, territoriale, de périphérie, des jeunes; comme ils ont de plus en plus souvent tendance à se retrouver entre semblables (à cause de la montée du racisme notamment), entre jeunes issus de l'immigration maghrébine, dans leur mobilité comme dans leur immobilité, ils adoptent un langage de frontière entre le français et l'arabe dialectal (le mot bonte, par exemple, est devenu dans leur langage courant la rchouma, ou la rachme; ou bien l'expression Dis oualla! ${ }^{8}$ qui signifie "jure-le par Allah") qui les situe de fait dans l'entre-deux, ni ici ni là, ni d'ici ni de là 9 . Il a fallu, lors d'ateliers d'écriture, faire comprendre à des collégiens que certains mots qu'ils écrivaient dans leur copie n'étaient pas compréhensibles par tout le monde et que, par conséquent, il fallait rédiger un glossaire à la fin de la rédaction pour aider le lecteur novice.

Leur histoire urbaine commune, leur même identité micro-territoriale a fait naitre entre eux des comportements de distinction sociale, par les attributs vestimentaires, mais aussi par la langue: le plaisir de maltraitter le français officiel appris à l'école (lieu du pliage à la norme), celui, policé, des adultes, mais aussi celui de la société des maîtres, signifie également la revendication de l'exclusion à travers un langage hermétique à ceux qui sont étrangers au groupe. C'est l'inversion d'un rapport de force sur le savoir (nous avons quelque chose entre nous auquel vous ne pourrez

7 Moi, enfant, je savais dire certains mots seulement en arabe et pas en fran:çais, comme par exemple le "gant de toilette"; je me souviens d'une anecdote qui m'était arrivée en classe de CE2 ou CM1; le maître avait demandé avec quoi on devait se frotter quand on se lavait et j'avais répondu avec "une kaissa " (un gant, en arabe dialectal algérien) et ma réponse avait beaucoup fait rire les élèves).

8 J'ai choisi cette expression comme titre d'un roman, Dis oualla, Paris, Fayard, coll. "Libres", 1997.

9 De la même manière, il faut remarquer lorsqu'ils se serrent la main pour se dire bonjour, ils portent la main sur le coeur comme le font traditionnellement les musulmans. 
68

pas accéder!), en même temps qu'une tentative de déplacer l'indigne, le Français de souche, dans le statut d'étranger, dans le rôle de l'Autre, le pas comme nous. C'est surtout par rapport à la police que ce sentiment et cette attitude sont les plus manifestes.

Dans ce contexte, l'utilisation par un membre du groupe d'expressions ou de formes langagières appartenant à la société centrale (l'école, les bourgeois, les "livres" ${ }^{10}$ ), équivaut à un signe de soumission à la société aliénante. De cette idée est née l'expression il parle comme un pédé, courante dans ce milieu, qui établit une analogie entre les codes du parler et ceux de la sexualité. Parler avec "des manières", en utilisant des mots de trop bonne tenue, des mots jugés déplacés par rapport à l'endroit où l'on est (le ghetto), se dit en opposition à la pratique qui consisterait à s'exprimer "sans aucune manière", c'est-à-dire sur un ton anarchique, vulgaire, violent, naturel, brut. Comme si dans un territoire où vit une population exclue, ou qui a le sentiment de l'être, le comportement linguistique devait afficher une culture d'opposition de principe, une émancipation ${ }^{11}$ sur le registre de la rebellion aux codes ${ }^{12}$. D'un point de vue psychosociologique, "parler avec des manières " prête à rire. On l'utilisera volontiers quand on veut caricaturer quelqu'un de "maniéré", en lui collant sur un ton ironique des expressions telles que "plaît-il"? ou "Qu'ouis-je"? En classe, comme dans son quartier, le jeune habitant de la cité défavorisée doit se tenir à distance d'une trop bonne maitrise et d'un bon usage de ces codes institutionnels pour ne pas être marginalisé dans son propre groupe.

Telles sont les ouvertures dans le champ psycho-sociologique que suscite l'usage d'une langue de la marginalité. Elles mettent en scène un individu en interaction permanente avec son groupe d'appartenance, d'où il puise certaines de ses valeurs identitaires.

10 "Il parle comme un livre..."

11 On pourrait faire des analogies avec la politique d'arabisation menée en Algérie par exemple, contre l'utilisation du français, langue du colonisateur.

12 Cf. Boris Seguin et Frédéric Teillard, Les céfrans parlent aux Français. Chronique de la langue des cités, Paris, Calmann-Levy, 1996. 


\section{Nous et je}

Sur le plan collectif, la langue des banlieues donne des indications sur "le positionnement du groupe" quand il n'est plus dans un cadre institutionnel établi dans la société. On ne s'exprime pas, lorsqu'on est ensemble dans le quartier, de la mêrne manière que lorsqu'on est en classe. Ce qui montre déjà la difficulté dans laquelle va se trouver un individu qui va choisir, en classe par exemple, de jouer le jeu de l'apprentissage normal de la langue et des autres codes de l'Éducation Nationale. Tôt ou tard, il devra choisir entre son appartenance au groupe (ses copains de quartier, ses "cousins") et son avenir personnel, en tant qu'individu. Ainsi, beaucoup d'enseignants de collège situés dans des quartiers sensibles pourraient confirmer ce constat: dans une classe de troisième ou de quatrième (quatorze ou quinze ans), l'élève qui voudrait poser une question sait à l'avance qu'il va prêter le flanc aux railleries, aux quolibets, d'une partie des autres élèves, a fortiori s'il utilise un français classique et bien traîté. C'est le principe d'auto-neutralisation.

Ce principe pourrait expliquer en partie pourquoi on remarque si souvent des élèves adolescents qui, pris individuellement, parlent vite, en broyant les mots avant leur sortie, de peur d'être compris, d'être pris en considération personnellement... d'être détachés de leur groupe, isolés. À cet égard, considérons l'engouement pour la musique rap. Le rythme musical où le très fort débit des mots (parlés ou chantés) prime par rapport au contenu, au sens de ce qui est dit, est tout à fait illustratif. De même, il est intéressant de remarquer comment l'articulation des mots est sacrifiée, remplacée en partie par un langage du corps.

À mon sens, "la langue des banlieues" témoigne d'un appauvrissement de la mâtrise des codes de la communication, en milieu urbain et dans les catégories sociales les plus défavorisées plus particulièrement. Elle est aussi le symbole du repli et de l'effacement de l'individu, de sa personnalité, face aux standards défensifs d'un groupe minoritaire.

Bien entendu, les conséquences sont préjudiciables à l'émancipation de l'individu, autant vis-à-vis de sa communauté que de son territoire. En effet, à la première occasion qui s'offrira au jeune d'aller en ville, de quitter sa réserve, son groupe familier, pour aller se présenter à une offre d'emploi par exemple, seul, face à un employeur qui va le juger par rapport à des signes 
extérieurs: les gestes, la tenue vestimentaire, la coupe de cheveux, l'état de la dentition, des ongles, la maîtrise de la langue... les risques d'échec sont majeurs. L'accent et les mots ethnicisés pourront constituer un élément disqualifiant pour le candidat. Ils signeront définitivement son adresse (déjà stigmatisée). À l'inverse, le jeune qui montrera ses capacités à acquérir les codes de la société, celui qui sera capable de ne pas laisser paraître, grâce à une bonne maîtrise du français, son origine (les marques de sa spécificité culturelle), aura plus de chance d'être intégré qu'un autre. Un usage déformé du français parlé "visibilise" l'individu à ses dépens.

Dès lors, la langue des banlieues me paraît constituer un phénomène circonstancié, territorialement et socialement, et incapable d'évolution. Ce que je dis aux jeunes des quartiers que je rencontre dans mes tournées littéraires, c'est que la meilleure façon de se défendre dans une société où l'on n'a pas trouvé sa place consiste à acquérir les règles du jeu social et à les utiliser pour une "promotion en interne". Parmi ces codes, la langue est le plus précieux. J'explique ainsi que si un jour, ils sont interpellés d'une manière offensante par un policier qui leur dirait: "Donne-moi tes papiers!", la plus percutante des répliques ne serait-elle pas: plaît-il? Chaque fois que je fais cette démonstration devant des jeunes des cités, réfractaires à l'idée de l'apprentissage du français, ils mesurent pleinement la fonction d'utilité sociale que revêt cet apprentissage lorsqu'il devient un instrument de reconnaissance de sa propre légitimité.

\section{Le rapport à l'oralité}

La pauvreté du langage est à mettre en résonance avec la pauvreté de la lecture dans les milieux défavorisés. Globalement, les enfants des quartiers en difficultés n'ont aucune proximité affective ou culturelle avec le monde des livres. Ainsi fais-je souvent l'expérience suivante avec des élèves de quatrième et de troisième, âgés de quatorze, quinze et seize ans. Je pose la question: Qui peut me donner le nom d'un écrivain français vivant? Systématiquement, trois types de réponses sont faites: d'abord Jean-Pierre Papin, un footballeur très populaire en Europe (il a publié un livre!) et Jean-Pierre Bellemare, un présentateur de télévision spécialisé dans les missions commerciales. Ensuite, Azouz Begag! C'est-à-dire l'écrivain qui est assis en face d'eux, 
vivant. Mis à part ces trois réponses, rares sont les noms d'écrivains français vivants que les élèves peuvent citer, au grand dam des professeurs de français qui assistent aux rencontres. Dans l'esprit des collégiens, la littérature et les livres renvoient à quelque chose de mort, de vieux et d'ennuyeux. Un écrivain vivant ça n'existe pas. Le livre est associé à la mort et l'écrivain au cimetière. Pour les enfants d'immigrés, encore plus que pour les autres, l'acte de lire en tant que tel requiert un effort inutile, dont on ne perçoit pas l'intérêt, a fortiori si les livres qu'on leur donne à lire n'ont guère de résonance sensorielle chez eux.

Comme dans mon histoire personnelle, pour la plupart, ces enfants d'immigrés sont issus de parents paysans, analphabètes ou illettrés; leur rapport à la chose écrite n'est pas socio-culturellement établi. Dans les familles maghrébines, la parole (ou le non-dit) est le principal véhicule de la communication. Rares sont celles dans lesquelles je suis allé qui ont dans leur appartement une bibliothèque ou seulement quelques livres. Lire des histoires aux enfants en bas âge, acheter des livres pour les fêtes ou les anniversaires sont des pratiques inconnues. On pourrait bien sûr transposer ce constat à beaucoup de minorités ethniques à faible niveau d'éducation et de revenus ${ }^{13}$. Dans ce type de familles, le rapport au livre est même vécu avec une certaine méfiance. Le livre renvoie en effet au caractère sacré de tout ce qui est posé comme inaccessible. Dans les familles maghrébines, le seul et vrai livre admis est celui des musulmans, le Coran; les autres, ceux de la connaissance, du savoir, de la curiosité intellectuelle sont réservés à une élite sociale. Par conséquent, il faut bien constater qu'à l'intérieur du monde des exclus sont érigées des frontières culturelles d'où les livres sont exclus d'emblée de l'évolution des enfants.

\section{Le livre, l'école et l'exclusion}

A "l'extérieur", dans l'école laïque française, les jeunes d'origine maghrébine ne peuvent pas entièrement se sentir comme des destinataires normaux du livre: ceux qui sont proposés par les "programmes scolaires" sont écrits pour les autres, pour "tous", c'est-à-dire les "Blancs", des enfants aux prénoms bien français,

13 Cf. par exemple, Alan Duff, Maori. The Crisis and the Challenge, New Zealand, Harper Collins, 1993, sur la situation des Maoris en Nouvelle-Zélande. 
avec des histoires de "blancs", des mots de blancs, des odeurs de blancs. Le contenu du livre n'a rien "d'impliquant" pour les élèves dont la visibilité sociale fait d'eux "des Noirs", des gens de couleur dans la France des Blancs.

Dans ces conditions, le rejet de la littérature classique est l'expression d'un refus plus profond, d'un réflexe défensif face à un monde dont ils ont le sentiment d'être exclus. Dès lors, le livre susceptible de les séduire doit soulever les "vrais" problèmes de ces jeunes dont les parents ne maittrisent pas la langue française. Ce livre doit parler des questions d'intégration, d'exclusion, de différence, de "moi " par rapport aux "autres", avec des mots justes, des mots exprimant le vécu de ces enfants d'origine maghrébine. Un tel livre, dans lequel on va même trouver des expressions arabes lexicalisées, touche l'enfant d'immigré à l'épicentre de ce qu'il vit, de ce qu'il sait, imagine et expérimente au milieu des siens, dans la société française. Comme tous les lecteurs, ce jeune attend d'un texte qu'il réfléchisse ses attentes et désirs, sa vie en "temps réel ", dans toute sa "brutalité" sensorielle.

Quelques livres qui répondent à ces exigences existent déjà en France. Ils se sont frayé une place entre Molière et Maupassant.

\section{Le roman « beur » comme littérature de proximité}

La littérature "beur" est née dans les années quatre-vingt ${ }^{14}$. Elle a constitué une nouveauté intéressante dans la production littéraire française. Arrivée à l'âge de vingt-trente ans, la première vague d'enfants d'immigrés maghrébins a produit ses premiers romans. Une douzaine de livres sont apparus sur la scène médiatique derrière une figure de proue: le livre de Mehdi Charef Le the au barem d'Archi-Ahmed (Mercure de France, 1983). C'est à partir de ce livre que la production romanesque des jeunes d'origine maghrébine de France a réellement pris son essor. L'écho médiatique que Le thé au barem d'Archi-Abmed a reçu au début des années quatre-vingt a démontré la curiosité du grand public

14 Voir les travaux du département de littérature maghrébine d'expression française de l'Université de Villetaneuse, dirigé par le professeur Charles Bonn et ceux du professeur Alec $\mathrm{G}$. Hargreaves (Immigration and Identity in Beur Fiction, op. cit.) 
pour ce genre de témoignage. D'autres auteurs se sont engouffrés dans le sillage. En 1984, Akli Tadjer publie aux éditions du Seuil Les A.N.I. du Tassili. En 1985, Nacer Kettane publie chez Denoël Le sourire de Brabim et Leilla Houari Zeïda de nulle part chez l'Harmattan. Pour la seule année 1986, six nouveaux romans apparaissent: Le Gone du Chaaba d'Azouz Begag, Point Kilométrique 190 d'Ahmed Kalouaz, Les "beurs de Seine de Mehdi Lallaoui, Palpitations intra-muros de Mustapha Raith, Georgette! de Farida Belghoul et L'escargot de Jean-Luc Yacine.

De quoi parlaient ces livres ${ }^{15}$ ? Sur le fond, il s'agissait essentiellement de la vie dans les HLM et les cités de transit de la banlieue parisienne en compagnie de copains d'infortune (Le thé au harem d'Archi-Abmed), des voyages au pays d'origine (LeS A.N.I. [les Arabes Non Identifiés] du Tassili, d'Akli Tadjer, Le sourire de Brabim de Nacer Kettane, Zeida de nulle part de Leïla Houari). Le racisme est aussi présent dans les récits comme celui d'Ahmed Kalouaz qui a raconté dans Point Kilométrique 190 le récit de la mort d'un jeune Algérien défenestré au début des années quatrevingt dans le train Bordeaux-Vintimille par des légionnaires en permission. Amar, le héros de Jean-Luc Yacine dans L'escargot est sur le point de recevoir un héritage inattendu, quand il meurt, victime d'un crime raciste, dans la région Nord-Pas-de-Calais. L'héroïne de Georgette! de Farida Belghoul est une fille de balayeur qui voue un amour immense et plein de tristesse à un père humilié quotidiennement, mais qui résiste avec fierté à sa vie difficile.

La problématique commune de ces textes est constituée de témoignages de vie où les héros sont souvent des jeunes d'origine maghrébine en conflit avec la société française. Mehdi Charef ainsi que Mustapha Raïth ont connu la prison. À titre personnel, je dois dire que c'est la lecture du Thé au harem de Mehdi Charef qui a provoqué en moi un tel choc que j'en ai acquis la certitude que je pouvais être aussi capable d'écrire un livre! Mon livre. C'est ainsi que j'ai commencé l'écriture de mon autobiographie $^{16}$. Mehdi Charef a été un pionnier, il a ouvert une brèche,

15 Mehdi Charef, Le thé au barem d'Arcbi-Abmed, Paris, Mercure de France, 1983; Leila Houari, Zeïda de nulle part, Paris, L'Harmattan, 1985; Farida Belghoul, Georgette!, Paris, Barrault, 1986; Ahmed Kalouaz, Point kilométrique 190, Paris, l'Harmattan, 1986; Nacer Kettane, Le sourire de Brabim, Paris, Denoël, 1985; Jean-Luc Yacine, L'escargot, Paris, l'Harmattan, 1986. Le Gone du Chaaba, Paris, Seuil, coll. "Point-virgule", 1986. 
74

rendu accessible la route vers les maisons d'édition. Le déclic occasionné par ce livre s'est manifesté chez moi par une totale identification, une projection dans les phrases de ce roman. C'était la première fois de mon existence d'enfant d'immigré en France qu'un livre m'offrait une telle possibilité d'identification tout à la fois communautaire, ethnique et sociale. Je me demandais comment cet auteur avait su écrire avec autant de justesse des choses si fortement et si secrètement enracinées dans ma personnalité. J'en riais de stupéfaction à chaque page tournée.

Aujourd'hui, c'est ce déclic que je cherche à favoriser chez les jeunes d'origine maghrébine que je rencontre dans les quartiers difficiles. Il est nécessaire d'en prendre conscience et de pouvoir l'utiliser pour déclencher des "libérations", des expressions, de la reconnaissance sociale. Dans les classes où je me rends depuis bientôt dix ans, je mesure à chaque occasion le caractère presque automatique de ce déclic. Dans les regards des élèves qui me dévorent des yeux et qui écoutent cet écrivain d'origine maghrébine, qui parle comme eux, qui dit parfois des mots en arabe, qui a vécu les mêmes conditions sociales qu'eux et dont on a étudié le livre en classe. On voit briller des lumières de magie, d'émerveillement, d'existence retrouvée. Fait symptomatique: combien de fois n'ai-je entendu ces élèves qui me demandent en catimini : M'sieur, tu parles arabe? Alors je réponds oui en arabe et ils jubilent de plaisir. Et quand les autres élèves, les "Blancs", leur font remarquer qu'on ne tutoie pas un écrivain, ils répondent aussi sec: Oui, mais celui-là il est à nous!

L'écrivain "beur" suscite une fascination. Il est vu comme un rescapé du monde de l'exclusion. Comment a-t-il pu s'en sortir? Par quel miracle a-t-il échappé aux pièges de la société? Comment est-il tombé dans l'univers des livres? Il n'est peut-être pas du même monde que nous... il est peut-être supérieurement intelligent... Sa venue en classe est une apparition, une faille dans les certitudes, dans le syndrome de l'échec scolaire qui touche beaucoup de ces jeunes.

\section{Le roman « beur » à l'école}

Dans les classes de collège, le roman "beur" devient un véritable outil pédagogique. Il assure la fonction d'un marchepied vers une littérature plus universelle, vers le livre d'une manière 
générale. Il est un acte préparatoire à la chose écrite. Par le biais de sa proximité avec l'univers culturel, social et urbain des jeunes issus de l'immigration maghrébine, il rend l'écrit vivant et lui donne un sens. La lecture devient ainsi prétexte à une comparaison de sa propre recherche identitaire par rapport à celle de l'auteur, à des questions sur les racines, sur la culture des parents. Autrement dit, la lecture permet un retour sur soi et c'est l'expérience de l'auteur qui rend cette introspection plus facile. Pour un élève d'origine maghrébine, la rencontre de l'un des siens est inouie. La lecture du roman "beur" en classe prend un aspect symbolique du plus haut niveau. C'est l'occasion pour ces élèves de retrouver une légitimité au sein de la classe, auprès du professeur et des élèves "blancs". À partir du moment où l'œuvre pénètre dans le cours de français, l'élève acquiert ses lettres de noblesse. "En reconnaissant l'œuvre, on reconnaît un vécu, une réalité, une identité. Le roman "beur" en classe, c'est une façon de penser l'intégration de l'élève dans une littérature, dans une école où il existe", a écrit un professeur de français d'un collège ${ }^{17}$. C'est ainsi que lorsque le livre contient des mots arabes, l'élève se fera un plaisir en classe d'en traduire pour les autres la signification en français. Les occasions ne sont pas si fréquentes pour lui d'apprendre aux autres quelque chose qu'il est le seul à connaître. L'introduction en classe d'un tel outil d'échange permet de réinstaurer une confiance du jeune en ses propres capacités de lecteur, de restaurer une fierté qu'il avait perdue.

Au-delà de cet avantage, dans les classes multiculturelles le roman "beur" peut également aider à évoquer et à penser la différence, une question d'actualité en France. L'un de ses atouts les plus notoires est sa capacité à dédramatiser les débats et les situations par l'humour. En se jouant de la différence, la dérision facilite en classe l'acceptation de l'autre par l'étude de son univers d'origine. Les très nombreuses lettres que je reçois régulièrement de la part d'élèves lecteurs et d'enseignants témoignent de cette évidence.

17 Stéphanie Kaickinger, Penser la différence, sa différence à partir d'une lecture suvie. Comment faire entrer en littérature une classe en difficulté par la médiation d'un roman beur, mémoire professionnel pour la titularisation, (sous la direction de Nadine Decourt), année 1994-1995. 
$\overline{76}$

\section{Conclusion}

Dans la littérature pour la jeunesse en particulier, c'est un euphémisme que de dire l'importance des messages éducatifs destinés à combattre le racisme et l'acceptation des autres. C'est un secteur sensible dans lequel il est indispensable d'habituer les enfants à la différence, à l'autre. C'est dans cette perspective qu'il me semble impératif d'impliquer dans les fictions des personnages qui reflètent la diversité de la population française. Bien sûr, des livres de ce type existent déjà avec des auteurs comme Susie Morgenstern, Thierry Lenain, Jean-Paul Nozires..., mais on peut les souhaiter plus nombreux. Quand on habite dans un quartier en difficultés, quand on est issu d'une famille pauvre, quand on est visible (d'origine africaine), quand l'école que l'on fréquente compte une majorité d'enfants d'immigrés, on se sent à côté des littératures pour la jeunesse dans lesquelles les héros s'appellent toujours Jean, François, Charlotte, Gervaise... Il est nécessaire de faire entrer dans le monde de la normalité le fait de s'appeler Ali ou Leïla et d'être en même temps Français, personnage d'une histoire de roman. De ce point de vue-là, le roman pour la jeunesse est un bon outil de lutte contre la xénophobie.

Le roman "beur" et, par-delà, le roman issu des minorités ethniques en France, mérite d'être encouragé, pour toutes les promesses qu'il offre dans une société multiculturelle. Malheureusement, la littérature "beur" n'est plus à la mode en France. On peut le déplorer. La grande majorité des auteurs qui ont publié des récits dans les années quatre-vingt en sont restés là. Seuls quelques-uns ont pu publier un second puis un troisième texte ${ }^{18}$. Ce qui est le plus surprenant, c'est que dans le domaine de la littérature pour la jeunesse, il y a seulement deux auteurs d'origine maghrébine: Malika Ferdjouk, qui publie des textes aux éditions Syros, et moi-même. Il y a matière à s'interroger sur une présence aussi faible des auteurs d'origine maghrébine dans ce secteur, vaste, qui doit beaucoup de son dynamisme économique aux politiques de développement de la lecture dans les milieux sociaux défavorisés.

18 Voir à ce sujet Alec Hargreaves, Immigration and Identity in Beur Fiction, op. cit., p. 173-182. 